\title{
The Correlation Between Distance Travelled And Spending
}

AUTHORS: Remington Free, Raphael Goldman-Pham, Maxim Vorobyov, and Devin Vyas SCHOOL: The Abelard School

\section{Abstract:}

The research was done for a high school competition where teams were given a data set of grocery store transactions and asked to pull interesting information out of the data. Our approach was to determine whether the approximate distance between the customer's home and the store had any impact on their spending, the hypothesis being that the greater the distance, the more the customer would spend over an extended period of time (three years). The reasoning behind this was that customers who travelled greater distances to reach a specific store would likely be motivated to buy more in order to justify the greater journey. The primary tools we used to test our hypothesis were Statistical Analysis Software (SAS) and Microsoft Excel. Our results obtained contradicted the initial hypothesis; it was found that shoppers who live closer to the stores spend much more than those who live further away.

\section{Key Words:}

Big Data, Inverse correlation, Customer spending analysis, Distance to store

\section{Background:}

The following research was conducted to submit to the Big Data Challenge, a high school computer programming and big data competition.1. Each team was given big data sets of thousands of entries and asked to find interesting information from the sets and come up with some conclusions.2. The data sets were store records from five Walmart stores across the Greater Toronto Area over three years, and included the following variables: year and week of transaction (2011-2013), customer ID number, cities/towns of customers, postal codes of customers, longitude and latitude of customers' homes, customers' gender, customers' date of birth, store ID, category of items (e.g.: Dairy, Beverages, etc.), number of transactions per category, quantity of items purchased per category, unique items per category, and total sales.

\section{Purpose:}

This project was an opportunity to learn about data analysis, and while we considered some alternate hypotheses suggested in the
SciNet presentation, we decided to focus our efforts on analyzing the correlation between the distance to the store and spending per customer. We set out to gain insight on the potential of big data analysis and learn whether or not it was possible to glean information about the preferences of certain demographics from our data set. Specifically, we hoped to determine whether or not it would be advisable for stores like Walmart to invest in advertising techniques which target those who travel greater distances to the stores (billboards on highways, expanded parking lots, etc.).

\section{Hypothesis:}

Our team decided to work with the distances between the customers and the stores. We hypothesized that as the distance between the two increases, so would the average spending of the customer. The thought behind this was: should a customer be more motivated to travel a further distance to come to a specific store, they may also be more motivated to purchase more while at the store.

\section{Procedure:}

Materials: The materials used were the computer programs Microsoft Excel, Statistical Analysis Software (SAS), online services such as Google Maps and Google Drive, and the data set provided by SciNet for use in this challenge.

Methods: Working with the data set provided for this challenge, we combined and integrated select data from separate files into a single workable file. Using SAS, this target data was then processed and transformed; we grouped the provided postal codes into Forward Sortation Areas (FSAs), which is the term used by Canada Post for the first three digits of a postal code (e.g.: M4V, M5T, etc.). This is used to represent a less specific geographical area than that of the postal code.3. In this process, 155 unique FSAs were found. This reprocessed data set was then manually merged with external data, taken from online service Google Maps, in order to find the driving distance in kilometres between the centre of each FSA and each store. Final analysis of this transformed data was performed using SAS and Microsoft Excel to calculate average spending per customer by distance and to visualize the results' correlations. 


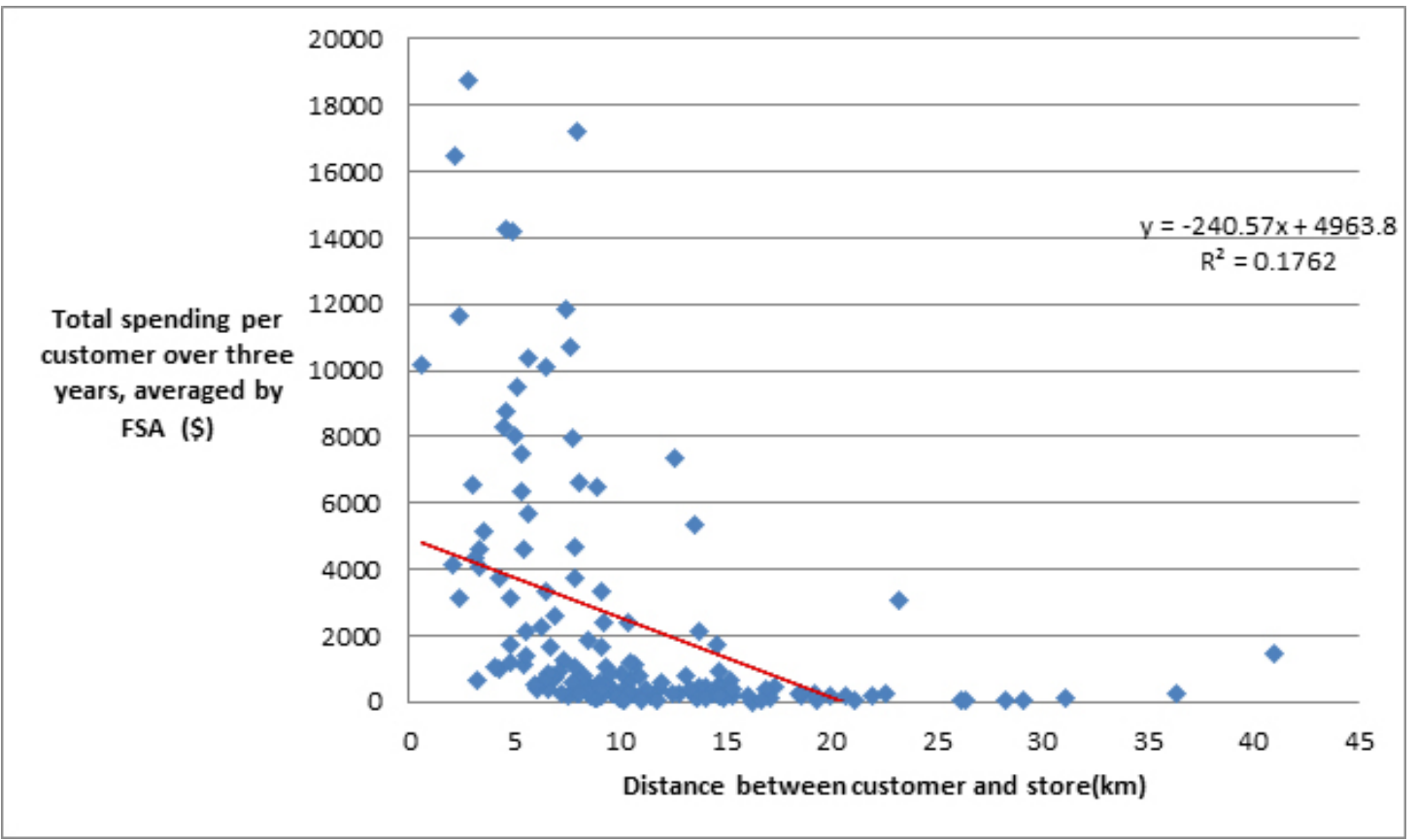

\section{Results/Observations:}

In looking for patterns, an inverse correlation was determined between the two variables, rather than a direct correlation as we originally anticipated. The first graph is a representation of the combined data from all the stores. Here, the distance is that between the center of the FSA and its corresponding store; for this reason, when making this graph, some FSA values were repeated as the customers from some FSAs frequented more than one store. It shows the overall inverse correlation between the distance to the store and the spending of the customer over this three year period, among all the stores. This first graph was a combination of the data from the following five. They each represent the correlation between distance and spending per customer for their respective stores.
Figure 1 represents the five stores overall. This linear model was used to emphasize the direction of this correlation, but the $\mathrm{R}$ squared coefficient is $17 \%$, meaning that only 17 percent of this data can be explained with this line, and shows that overall in the five stores the data is not homogenous. The coefficient near $x$ is negative, so there is an inverse correlation, which is equal to $42 \%$. The coefficient after $x$ shows the amount a person would spend if he lived zero kilometers away from the store. While the greatest distance found was $41 \mathrm{~km}$ (with a corresponding spending value of $\$ 1,462$ ), the smallest distance was $0.6 \mathrm{~km}$ (with a corresponding spending value of $\$ 10,180$ ).

The graph can be seen broken down into the individual stores:

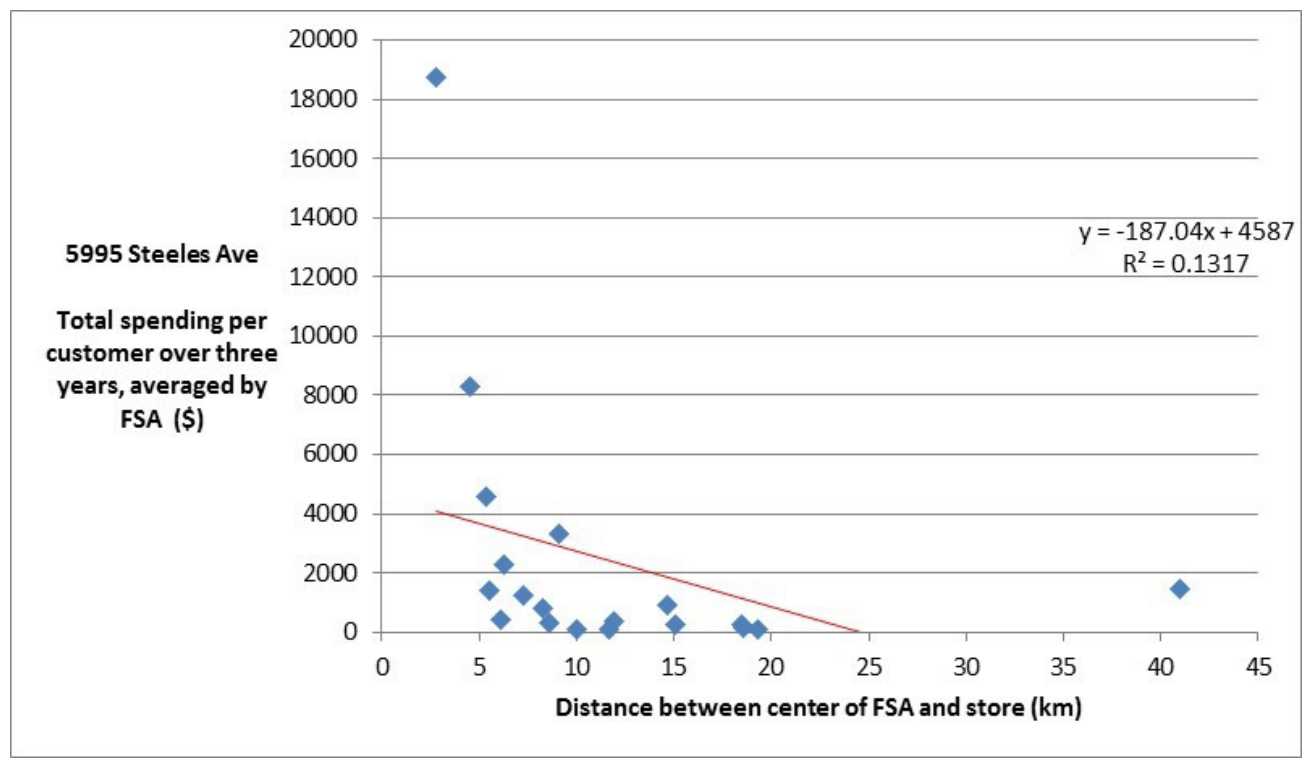



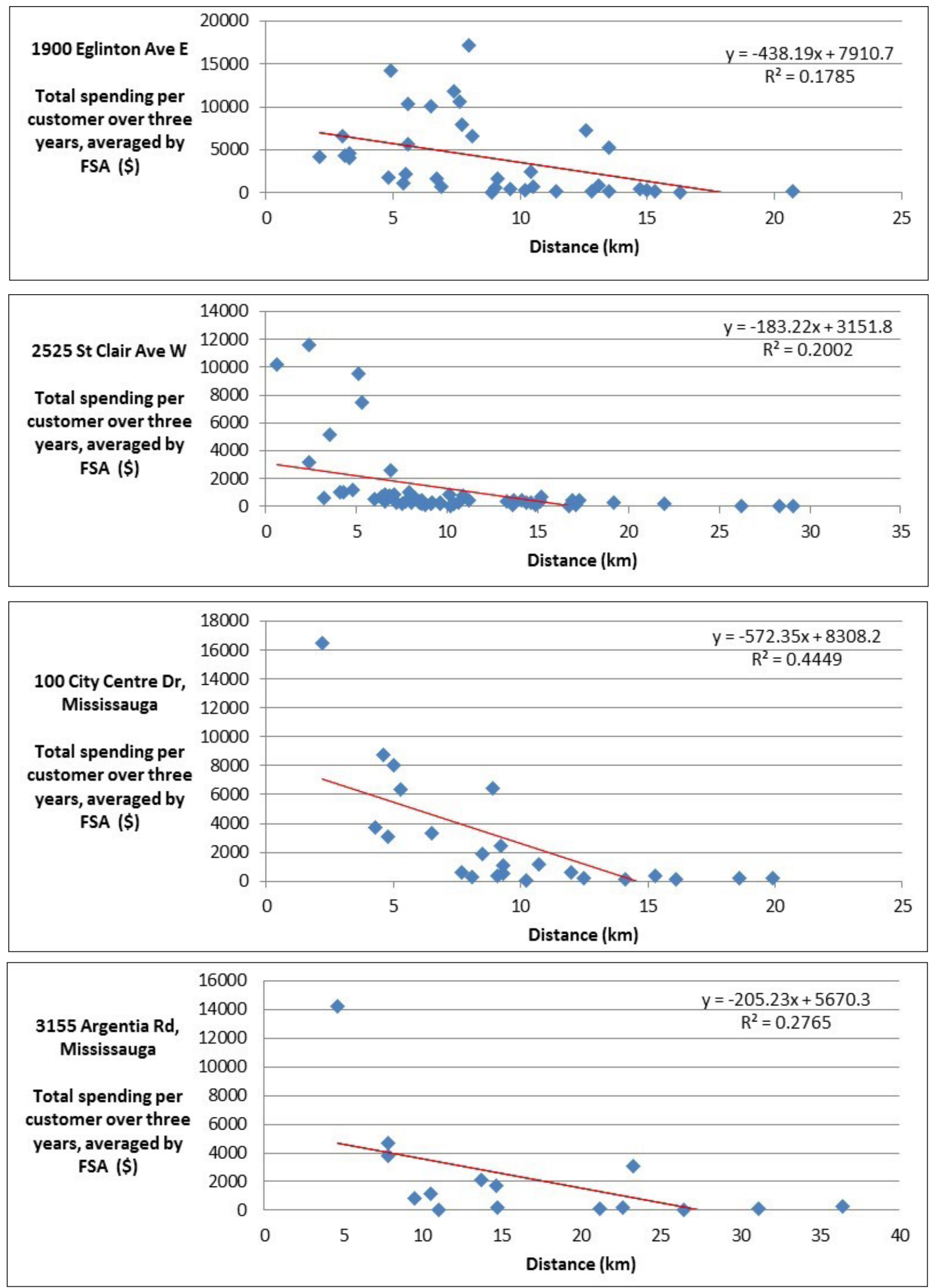


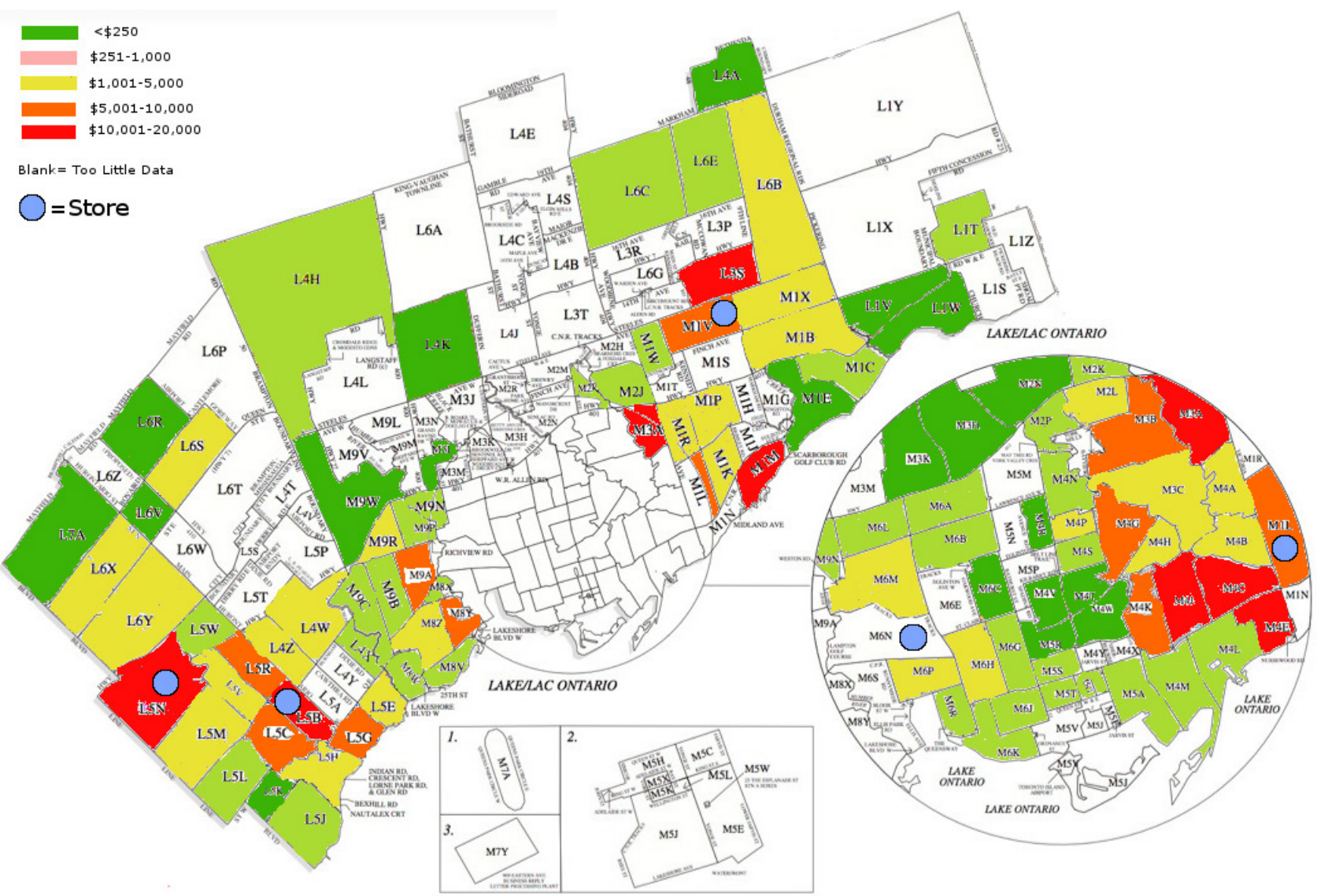

Figure 7 is a map of the Greater Toronto Area, labelled based on average spending per customer over the three year period, organized by FSA. The five stores are represented in blue circles on the map. White areas on the map represent FSA for which we had little or no information.

\section{Conclusions:}

The results were notable because they were in fact exactly the opposite of what was initially predicted. Instead of confirming our hypothesis that the shoppers who were further away would spend more on a trip due to the increased commitment of travelling a long distance to reach that particular store, the opposite occurred. As shown in the graphs as well as the visual representation (Figures 1-7), the highest spending areas were those closest to the stores. This may be because individuals living close to the store may make frequent trips to the store for immediate needs (e.g.: milk, paper towels) that may add up to cost much more than the money spent by those few who travel a long distance. While we were satisfied with this interpretation in relation to our original hypothesis, further research into this topic could be telling in determining whether there was a correlation between the distance and the amount spent per visit to the store, as our work only looked at the spending over a three year period. It may also be valuable to note that customers who lived further away from a store may have been shopping at that particular store only because they happened to be in that part of town. Another factor that is relevant to the results is the average household income of the FSAs observed. After some research on Toronto Census, it was discovered that the lower spending was not due to a lower income: in fact, the areas with the lowest average household income were the highest spenders at these stores, likely because Walmart and similar stores are marketed towards a clientele with a lower household income. In accordance with our original goal to determine the best advertising techniques for stores like Walmart, It would be advisable to work towards building customer loyalty in nearby areas and continue marketing for customers with a lower household income.

\section{Appendix}

Acknowledgements: We would like to thank Dr. Sasha Noukhovitch, and the organizers of the Big Data Challenge for inspiring this research. Special acknowledgements must also be made to our teacher and mentor, Dr. Allan Randall, and our principal, Ms. Michelle Lefolii. 


\section{References:}

1. Big Data Challenge: Competition for high school students. iEcarus [Online] Available from: http://iecarus.com/trainings-and-events/big-data-challenge/

2. BigDataChallenge2014. SciNet Wiki. [Online] http://wiki.scinethpc.ca/wiki/index.php/ BigDataChallenge2014

3. Glossary. Canada Post. [Online] Available from: http://www.canadapost.ca/tools/pg/glossary- e.asp\#F for definition of FSA

4. WellbeingToronto Web App. Toronto. [Online] Available from: http://map.toronto.ca/wellbeing/

\section{REVIEW}

\section{by Dr. Marcelo Ponce,}

\section{SciNet, University of Toronto}

In this paper the authors studied the correlation (if any) between the distance travelled by customers and their spending patterns: they model an underlying relation between distance and spending habits by proposing a working hypothesis, and testing it. By doing so, they not only unveiled the validity of the paradigm initially proposed but learned more about the data itself, making sense of the data and their own results. I must emphasize that it is a well written article, easy to read and clearly presented. However, I should mention that the plots and figures are a bit disconnected from the text. As a way to improve this article, I would suggest as a general rule, that there shouldn't be figures that are not cited and discussed within the text. All plots must have captions self-describing the respective figure. Finally, each reference included should be properly mentioned in the paper where it actually belongs.

The authors have used a great variety and diversity of tools, which is of great importance in dealing with real problems. This represents a mature and solid article that contains all the main elements of a professional scientific report. Furthermore, it is interesting to emphasize (as these students have discovered), that real world data, and even more specifically, models trying to reflect the trends in complex situations, are usually more complicated and intricate than our assumptions. 